\title{
Statistical studies of flare stars and other flashing objects carried out at the Byurakan Observatory
}

\author{
A.A. Akopian * \\ Byurakan Astrophysical Observatory, Armenia
}

\begin{abstract}
The review briefly presents the statistical studies of flare stars and related objects carried out at the Byurakan Astrophysical Observatory, in particular: i) determination of the evolutionary status of flare stars, ii) an explanation of the observed difference between flare stars of the galactic field (type UV Cet) and flare stars of systems (open clusters, associations), iii) the connection between flare stars and T Tauri stars, iv) short-term and long-term (evolutionary) variability of flare activity, v) original statistical methods developed for the study of flare stars and their systems, vi) recent advances in research on flashing objects. This review does not present such important areas of research on flare stars as the determination of physical parameters, photometry and colorimetry of stars and their flares, detailed studies of individual stars, theoretical works on possible mechanisms of flares. These areas were well presented in many books and reviews by Ambartsumian and Mirzoyan.
\end{abstract}

\section{Introduction}

In the first years after the founding of the $\mathrm{BAO}$, Ambartsumian's main research was related to the origin and development of celestial bodies. Analysis of the accumulated observational data allowed him in 1947 to discover in the Galaxy new type stellar systems, which he called "stellar associations". He established the existence of two types of associations - OB and T and predicted that they are dynamically unstable systems and have a relatively recent origin. The prediction by Ambartsumian $(1947,1949)$ about the dynamic instability of stellar associations and their expansion was confirmed as a result of the analysis of proper motions of associations stars, carried out in Leiden observatory by Blaauw and coworkers.

This led to the inevitable and fundamental conclusion about the continuous process of star formation at the present stage of the development of the Galaxy. This was a refutation of the concept of the simultaneous origin of stars in the Galaxy. This work indicated the fundamental possibility of observing and studying the development of stars and opened the door for astronomical studies of the evolution of stars and stellar systems, which seemed almost impossible within the framework of the old ideas about the origin of stars.

Ambartsumian drew attention to unusual continuous radiation - another feature of the physical nature of members of stellar associations, observed, in particular, in stars of the T Tauri and UV Ceti type/flare stars, as well as in cometary nebulae, and to the nonthermal origin of this continuous emission. The flare activity of UV Ceti type/flare stars is one of the most characteristic features of the red dwarf stars at the early stages of evolution. The radiation emitted from a UV Ceti type/flare star during the flare, which occurs between the long periods of the quiescent state of the star, shows physical similarity with the radiation of $\mathrm{T}$ Tauri type stars. But even during the quiescent state, the radiation spectrum displays some signs of chromospheric activity.

The physical similarity between the radiation of flare stars and rapid variations of the brightness of the T Tauri type stars (Ambartsumian, 1954), testifies the fact that in both cases similar processes of radiation take place, different from blackbody thermal radiation, at least during the flares. It is in itself an indication of the close genetic relationship between these two classes of stars. This conclusion has been confirmed by the investigations of Haro and coworkers (Haro, 1957, 1964). This aroused great interest in the origin of this entirely new phenomenon. Up to now, extensive studies of these objects are underway both at the Byurakan Observatory and the other observatories. Observations and statistical studies of flare stars strongly were stimulated when it had been shown by Ambartsumian (Ambartsumian, 1969, Ambartsumyan et al., 1970$)$ that in a relatively young stellar aggregate of Pleiades $\left(\approx 5 \cdot 10^{7}\right.$ years) almost all the red dwarf stars, fainter than a certain absolute magnitude, must be flare stars.

*aakopian57@gmail.com 
Comparing the estimated number of flare stars with the available estimates of the total number of stars in the Pleiades led to the conclusion that the flare star stage (the stage of flare activity) is regular in the evolution of red dwarf stars, through which all of these stars pass. These results were so important for the evolution of red dwarfs that they initiated wide-field photographic observations at the observatories of Asiago (Italy), Byurakan (USSR, Armenia), and Tonantzintla (Mexico) to seek out and study flare stars in the regions of different ages stellar clusters and associations, and these studies were later undertaken at Abastumani (USSR, Georgia), Konkoly (Hungary), and Rozhen (Bulgaria). Besides the Pleiades system, other systems of flare stars, especially the systems of Orion, Praesepe, Hyades, etc., were studied in the international program. These observations led to the discovery of a large number of new flare stars and showed that they were unusually abundant in these systems. The regular observations of flare stars systems stopped in the early $80^{t h}$ of the $20^{t h}$ century because of cardinal changes of types of astrophysical detectors. These efforts ultimately yielded results that were important for understanding the development and evolution of red dwarf stars.

This review briefly presents the statistical studies of flare stars and related objects carried out at the Byurakan Observatory, in particular: a) determination of the evolutionary status of flare stars, b) an explanation of the observed difference between flare stars of the galactic field (type UV Cet) and flare stars of systems (open clusters, associations), c) the connection between flare stars and T Tauri stars, d) short-term and long-term (evolutionary) variability of flare activity, e) original statistical methods developed for the study of flare stars and their systems, f) recent advances in research on flashing objects. The basis for these studies was the data set of observations of flare stars obtained as a result of the collaboration of the Byurakan Observatory with other observatories.

This review does not present such important areas of research on flare stars as the determination of physical parameters, photometry and colorimetry of stars and their flares, detailed studies of individual stars, theoretical works on possible mechanisms of flares. These areas were well presented in many books and reviews by Ambartsumian and Mirzoyan.

\section{Observations and observers/researchers}

Observations of flare stars carried out in the Byurakan observatory can be combined into three groups: photographic, photoelectric and spectral.

\subsection{Photographic observation}

1) Main task - detection of the possible large, statistically significant number of flare stars and their flares in different systems of flare stars, simultaneous photometric observations of flare stars, their statistical, photometric and colorimetric studies.

2) Main targets - flare stars of star clusters and associations - the Pleiades, Orion association, NGC 7000 (Cygnus), Praesepe, Hyades, Taurus Dark Clouds

3) The telescopes used $-1 \mathrm{~m}$ Schmidt telescope (field of view is $4.1^{\circ} \times 4.1^{\circ}$ ) and $53 \mathrm{~cm} \mathrm{Schmidt}$ telescope (field of view is $5^{\circ} \times 5^{\circ}$ ) with the Kodak plates $16 \mathrm{~cm} \times 16 \mathrm{~cm}$ as detectors.

4) The method of detecting flare stars was a "chain" method, when several images of a star are obtained on a plate, each with an exposure of up to 10 minutes. Its alternative was the "tracks" method proposed by Chavushian, which is more informative but it was possible to apply only to a small sample of bright flare stars. The "track" method was rarely used.

5) Photometric bands - the majority of observation have been carried out in Johnson's photometric bands, mainly in the B band, allow to detect more flare stars and flare than in $\mathrm{U}$ and $\mathrm{V}$ bands.

6) The main observation period was the 70th of the last century. A significant part of the Byurakan observatory staff, headed by Ambartsumian and Mirzoyan, head of the Department of Physics of Stars and Nebulae, were involved in the observations. A group of astronomers of the Department of Physics of Stars and Nebulae (headed by Parsamian E.S.) actively collaborated with Mexican astronomers to study Orion flare stars and related objects. Here are the observers/researchers who participated in the studies: Chavushian, Erastova, Kazarian, Gasparian, Garibjanian, Hambarian, Melikian, Ohanian. Foreign astronomers also were involved in these observations and studies.

\subsection{Phototelectric observation}

1) Main task and targets - observation of UV Ceti, BY Dra type flare stars of solar vicinity with high photometric accuracy.

2) The telescopes used - $50 \mathrm{~cm}$ and $40 \mathrm{~cm}$ Cassegrain telescopes, $2.6 \mathrm{~m}$ telescope

3) Photometric bands - the majority of observation have been carried out in Johnson's photometric bands UVB. 
4) The main observation period was the 70th and early 80th of the last century. Group members Oskanian V.S.(head of group), Melkonian A.S., Oskanian A.A. Jr.

5) Important results - The expressing the sequence of flares of individual stars via Poisson law by Oskanyan \& Terebizh (1971), based on the study of the longtime series of photoelectric observations of some flare stars in the vicinity of the Sun.

\subsection{Spectral observation}

Spectral observations began on the 80th of the 20th century. In contrary to photographic and photoelectric observations, spectral observations of flare stars are continued up to now.

1) Main task and targets - spectral observation of UV Ceti type flare stars of solar vicinity and their flares, discovering new flare stars in star-forming regions, detailed study of the physical characteristic of flare stars and their flares

2) The telescope used $-2.6 \mathrm{~m}$ telescope

3) The members of the Department of Physics of Stars and Nebulae were involved in the spectral observations (Mirzoyan, Parsamian, Garibjanian, Melikian, Hambaryan, Ohanian). After the structural reformation of the observatory, the spectral observations continued by Melikian and his coworker A. Karapetian. Foreign astronomers also were involved.

\section{Statistical studies of flare stars and related objects. Methods, applications and obtained results}

In this section statistical methods developed at the Byurakan Observatory by Ambartsumian and followers for the study of flare stars and related objects are presented, in particular for the:

1) estimation of the unknown number of flaring objects,

2) determination the distribution function of the frequency of the flares,

3) study of the short- and long-time (evolutionary) variability of flare activity,

4) determining the spatial distribution of flare stars in the parent systems.

The basic assumption used in a) - c) is the Poissonian character of the flares sequence, both as with constant and variable parameter (intensity) of the Poissonian process.

\subsection{Estimators of the unknown number of flare objects}

Flare stars in stellar systems. Ambartsumian estimator Estimation of the total number of flare stars in the Pleiades has been obtained (Ambartsumian, 1969) under two following assumptions:

1) The sequence of flares at each flare star represents a random Poissonian process.

2) All flare stars of the given system have the same mean frequency of flares.

Ambartsumian estimator allows to estimate the number $n_{0}$ of flare stars flares of which have not been registered yet, via the known numbers $n_{1}$ and $n_{2}$ of stars which already showed, accordingly, one and two flares, as follows:

$$
n_{0}=\frac{n_{1}^{2}}{2 n_{2}}
$$

It should be noted that the first assumption is quite well-founded (Oskanyan \& Terebizh, 1971). As to second assumption, one can give it up. Chavushian (1979) confirmed the heterogeneity of Pleiades cluster flare stars flares frequency. In this case, the number of stars in the system for which $k$ flares each is observed over time $t$ is equal to

$$
n_{k}=N \int \varphi(\nu) \frac{(\nu t)^{k} e^{-\nu t}}{k !} d \nu
$$

Ambartsumyan et al. (1970) using by Cauchy-Schwarz inequality have shown that when second assumption is incorrect, one can derive only the lower bound of the number of flare stars ${ }^{1}$. The upper

\footnotetext{
${ }^{1}$ Now Ambartsumian's estimator widely used in various branches of statistics, medical and social sciences, biology and ecology, linguistics, criminology, epidemiology, cryptography, etc. In these branches, this method, or closely related methods, are named in different ways: capture-recapture, mark-recapture, capture-mark-recapture, mark-recapture, sight-resight, markrelease-recapture etc.
} 
bound of the flare stars number has been estimated in the assumption that the density of distribution of frequencies of flares is too slowly decaying exponential. Ultimately, the following inequality holds:

$$
\frac{n_{1}^{2}}{n_{2}}>n_{0}>\frac{n_{1}^{2}}{2 n_{2}}
$$

The application of this estimator to the Pleiades, based on observations by Ambartsumian \& Mirzoyan (1971), Ambartsumyan (1970), Ambartsumyan et al. (1972, 1973), led, in particular, to the following conclusions Ambartsumian \& Mirzoian (1975), Ambartsumyan et al. (1971):

1) The number of flare stars showed flares with a photographic amplitude of more than $0^{m} .6$ are observed, is about a thousand.

2) The mean flare rates for different flare stars in the Pleiades differ, but most of them experience, on average, one photographic flare every 3600 hours.

3) At a lower luminosity in the normal state outside the flares (at the minimum brightness), the average frequency of the observed flares is higher.

Comparing the estimated number of flare stars with the available estimates of the total number of stars in the Pleiades led to the above-mentioned conclusion that the flare star stage (the stage of flare activity) is regular in the evolution of red dwarf stars, through which all of these stars pass.

Table 1 gives the summary data for the two most studied systems, where $T$ is the effective time of photographic observations, $n$ - the number of discovered flare stars $n^{\prime}$ - number of registered flares, $n_{1^{-}}$ the number of flare stars for which one flare was recorded, $n_{2}$ - the number of flare stars for which two flares were recorded, $N$ - the estimate of the total number of flare stars, $t$ - the mean discovery time of a new flare star, and $r$ - the distance of the system (which were adopted in early studies).

Besides the estimation of flare stars number, also the relative number of $\mathrm{T}$ Tauri stars with flaring activity in the Orion association and other systems (e.g Ambartsumyan, 1970)(were estimated. It was shown that about $1 / 4$ of Tauri stars in this system can produce "photographic" flares. This led, along with the arguments given by Haro $(1957,1964)$, to the conclusion that the flare star stage begins shortly before the end of the $\mathrm{T}$ Tauri stage and for a short time the star shows flare activity along with the $\mathrm{T}$ Tauri type activity. Now the genetic and evolutionary connections between T Tau and UV Cet stars appear to be more complex, but there is no doubt about their close relationship.

The Ambartsumian estimator can be used in the studies of other randomly flashing objects. As an example, in a statistical study of a sample of 1547 superflares of 270 solar-type stars found during an analysis of data on more than 80000 stars of the solar-type (obtained by the Kepler orbital observatory during the first 500 days of observations), the total number of stars capable of superflares and the superflare distribution frequency function were determined (Akopian, 2017). One may conclude that the number of stars capable of superflares is roughly $0.5 \%$ of the total number of all the solar-type stars that were studied. This most likely indicates that solar-type stars with superflares either have some rarely encountered feature(s) or are in a short-duration phase of their evolution. Unavoidable observational errors, incorrect classification of the stars, etc., can hardly affect the main conclusion that the overwhelming majority of solar-type stars cannot produce superflares over a time comparable to the lifetime of these stars.

Table 1. Results of photographic observations of flare stars

\begin{tabular}{|l|l|l|l|l|l|l|l|l|}
\hline System & $T(\mathrm{hrs})$ & $n$ & $n^{\prime}$ & $n_{1}$ & $n_{2}$ & $N$ & $t(\mathrm{hrs})$ & $r(\mathrm{pc})$ \\
\hline Pleiades & 3200 & 546 & 1495 & 287 & 92 & 995 & 5.9 & 125 \\
Orion & 1600 & 491 & 654 & 380 & 76 & 1441 & 3.3 & 500 \\
\hline
\end{tabular}

Important mathematical and statistical properties of Ambartsumian estimator Methods for the statistics of flare stars developed mainly at the Byurakan Observatory and originate in papers by Ambartsumian (1969), Ambartsumyan (1978). In terms of their scientific value and novelty, the mathematical methods developed by Ambartsumian for solving specific astrophysical problems in the statistics of flare stars are just as significant as the astrophysical results obtained using these methods. At a conference in honour of his sixtieth birthday in 1968. Ambartsumian (1969) gave a talk in which he proposed a clever and unexpectedly simple means of estimating the number of flare stars in stellar systems (Eq 1.) The most important mathematical and statistical properties of Ambartsumian estimator are:

1) Ambartsumian estimator gives the lower bound of the number $n_{0}$

2) Ambartsumian estimator is robust one. This important result was obtained by who has shown, that among possible estimators of type

$$
n_{0}(t)=\frac{n_{k}(t) \cdot n_{l}(t)}{n_{m}(t)} \cdot c_{k, l, m}(t)
$$


where the factor $c_{k, l, m}(t)$ generally depends on the kind of density of distribution of mean frequency of flares, robust estimators are those for which equality $k+l=m$ takes place; i.e. the estimator (??) is robust. According to (Ambartsumian, 1998) another robust estimator is

$$
n_{0}(t)=\frac{n_{1}(t) \cdot n_{2}(t)}{n_{3}(t)}
$$

1) Variation of $n_{0}$ has the following form (Bohning, 2010):

$$
\sigma_{n_{0}}^{2}=\frac{n_{1}^{3}}{n_{2}^{2}}\left[1+\frac{1}{4} \frac{n_{1}}{n_{2}}\left(1-\frac{n_{2}}{N_{o b s}}\right)\right]
$$

where $N_{o b s^{-}}$a number already discovered flare stars.

1) The mean flare frequency can be determined from the expression $\langle\nu t\rangle=2 n_{2} / n_{1}$ (Ambartsumian, $1969)$ is a maximum likelihood estimate for the doubly truncated Poisson distribution in which no events with $k=0$ and $k \geq 3$ are observed (Akopian, 1998).

Alternative estimators Ambartsumian's results stimulated works in this area. Several new estimators of the number of flare stars were published. The following estimator was obtained by Mirzoyan et al. (1977):

$$
\begin{aligned}
& N=\frac{\sum_{k=1} n_{k}}{1-e^{-\langle\nu t\rangle}}, \\
& \frac{\langle\nu t\rangle}{1-e^{-\langle\nu t\rangle}}=\frac{n}{N_{1}}
\end{aligned}
$$

where $\langle\nu t\rangle$ is the solution of the second, transcendental equation of the equation system $\operatorname{Eq}(7), N-$ the total number of flare stars, $n$-total number of flares.

The estimate (7) was derived later in Akopian (1998), where other estimators based on a Poisson distribution also were suggested. The well-known estimators of the parameter $\nu t$ truncated at the point $k=0$ of the Poisson distribution were used. Estimators of the number of flare stars were obtained by assuming that these estimators are equal to the estimates for the non truncated Poisson distribution. Of the proposed estimates, the following estimator merits attention:

$$
n_{0}=n_{1} \frac{\sum_{k=1} n_{k}}{\sum_{k=2} k n_{k}}=n_{1} \frac{N_{o b s}}{\sum_{k=2} k n_{k}}, \quad N=\frac{\sum_{k=1} n_{k} \sum_{k=1} k n_{k}}{\sum_{k=2} k n_{k}}=\frac{n N_{o b s}}{\sum_{k=2} k n_{k}}
$$

This estimate also gives a lower limit on the number of unknown flare stars (Akopian, 1998).

The problem of predicting the number $n_{r}(t)$ of flare stars, based on the data known for the observation time T, was solved by Mnatsakanyan (1986) and Mnatsakanyan \& Mirzoyan (1988). According to the latter,

$$
\begin{gathered}
n_{r}(t)=\sum_{k=r}^{\infty} n_{k}(T) \cdot C_{k}^{r}\left(\frac{t}{T}\right)^{r}\left(1-\frac{t}{T}\right)^{k-r}, \quad r=0,1 \ldots \\
N_{\text {obs }}(t)=\sum_{k=1}^{\infty} n_{k}(T) \cdot\left[1-\left(1-\frac{t}{T}\right)^{k}\right]
\end{gathered}
$$

The detailed history of the Ambartsumian estimator and its analogues was given in Akopian (2018).

\subsection{Determining the distribution function of the frequency of flares}

Ambartsumian method. If each flare star is characterized by a mean frequency $\nu$, then a cluster as a whole will be described by a distribution of the mean frequencies over the set of flare stars contained in it. Let us denote the normalized density of this distribution by $\varphi(\nu)$. Up to now, it has been essentially impossible to determine $\varphi(\nu)$ by direct calculation because of the small number of detected flares for individual stars. In Ambartsumyan (1978) proposed a statistical method for determining $\varphi(\nu)$ based on solving the inverse problem which avoided this difficulty. To do this he used a function $m_{i}(t)$ that gives the number of stars for which the $i$-th flares were observed during the interval from $t$ to $t+1$. The probability of this event is equal to the product of the probability that exactly $(i-1)$ flares have occurred up to the moment $t$ by the probability that a flare will be detected in the interval from $t$ to $t+1$. Hence, for a Poisson process, the function $m_{i}(t)$ is given by

$$
m_{i}(t)=N \int \nu \frac{(\nu t)^{i-1}}{(i-1) !} e^{-\nu t} \varphi(\nu) d \nu
$$

In particular

$$
m_{1}(t)=N \int \nu e^{-\nu t} \varphi(\nu) d \nu
$$


will be the number of new flare stars of the given aggregate which are discovered per unit time, for only when a flare is observed can a star be included among the flare stars. Note that the number $m_{1}(0)\left(m_{0}\right.$ precisely its mathematical expectation) is also equal to the mean number of all flares in the aggregate per unit time:

$$
m_{1}(0)=N \nu_{m}=N \cdot \frac{n(t)}{t}
$$

where $\nu_{m}$ is the mean of the mean frequencies of the flares in the set of stars and $n(t)$ is the total number of flares detected up to the time $t$.

Since evolutionary effects appear only after hundreds of thousands or millions of years, the physical state of the entire sample of flare stars can be considered practically unchanged throughout all our observations. Thus, $m_{1}(0)$ will characterize the density of flares in time, both at $t=0$ and throughout the entire observation period. The mathematical expectation of $m_{1}(t)$ should fall off monotonically, for flares of already discovered stars are not counted in the calculation, since $m_{1}(0)$ denotes the number of flares per unit time of stars that have not been discovered up to time $t$, the number of which will fall off with increasing $t$. It follows directly from Eqs. (12) and (13) that the mathematical expectation of $m_{1}(t)$ is fully determined by the distribution $\varphi(\nu)$ of the frequencies and the following simple relationship exists between them:

$$
\frac{m_{1}(t)}{m_{1}(0)}=\frac{\int \nu e^{-\nu t} \varphi(\nu) d \nu}{\int \nu \varphi(\nu) d \nu}=\frac{\int \nu e^{-\nu t} \varphi(\nu) d \nu}{\nu_{m}}
$$

so the question of determining $\varphi(\nu)$ reduces to taking the inverse Laplace transform of the observed function $m_{1}(t) / m_{1}(0)$ :

$$
\varphi(\nu)=\frac{\nu_{m}}{\nu} L^{-1}\left[\frac{m_{1}(t)}{m_{1}(0)}\right]
$$

where $L^{-1}$ - is the inverse Laplace transform operator. Since the observation of the first flare of a star is the discovery of a new flare star, it follows that the chronology of the discoveries is the input data for determining the distribution of the flare frequency.

The function $m_{1}(t) / m_{1}(0)$ is subject to strong fluctuations, which are inevitable in a once-observed realization of this function so that preliminary smoothing is necessary when searching for a solution. Eq. (11) implies that the mathematical expectation of the numbers $m_{i}(t)$ and $m_{i+1}(t)$ for the same stars are related as follows:

$$
\frac{d m_{i}(t)}{d t}=\frac{(i-1) m_{i}(t)}{t}-\frac{i \cdot m_{i+1}(t)}{t}
$$

In particular, for $i=1$ :

$$
\frac{d m_{1}(t)}{d t}=-\frac{m_{2}(t)}{t}, \quad m_{1}(t)=m_{1}(0)-\int_{0}^{t} \frac{m_{2}\left(t^{\prime}\right)}{t^{\prime}} d t^{\prime}
$$

In this case, because the observed function is under the integral sign, the resulting $m_{2}(t)$ are much less susceptible to fluctuations than are those directly determined "by counting detections" in a single realization. Eq. (17) makes it possible to obtain a more reliable "detection" curve based on the chronology of second flares than that obtained from the chronology of detections. Let to note yet another, very simple to apply, but rarely used method for smoothing the function $m_{1}(t)$. It is easy to see that Eqs. (2), (12), and (13) imply that

$$
\frac{m_{1}(t)}{m_{1}(0)}=\frac{n_{1}(t)}{n(t)}
$$

where $n(t)$ is the total number of flares detected up to $t$ and $n_{1}(t)$ is the number of singly flaring stars. For the Pleiades cluster, where about 500 flare stars are currently known, calculations have shown that the values $m_{1}(t)$ given by Eq. (17) yield a smooth curve that is closely coincident with the smoothed curve of $m_{1}(t)$, determined by direct calculation. Both results are in satisfactory agreement with the assumption that $m_{1}(t)$ has the form

$$
\frac{m(t)}{m(0)}=\frac{1}{(1+a t)^{b}}
$$

where $a=0.0026$ and $b=2 / 3$.

Ambartsumian proposed the following algorithm for determining the probability density of the mean flare frequencies $\varphi(\nu)$ :

1) The function $m_{1}(t) / m_{1}(0)$ is computed by direct calculation,

2) The function $m_{2}(t)$ is calculated and Eq. (17) is used to smooth the function $m_{1}(t) / m_{1}(0)$.

3) The inverse Laplace transform based on Eqs. (14) (15) is used to determine $\varphi(\nu)$. 
The correctness of the resulting solution $\varphi(\nu)$ was verified using other observational data obtained independently of $m_{1}(t)$ and $m_{2}(t)$. In particular, knowing $\varphi(\nu)$, the ratios $n_{k} / n_{1}=\int \varphi(\nu) \frac{(\nu t)^{k} e^{-\nu t}}{k !} d \nu / \int \varphi(\nu) \nu t e^{-\nu t} d \nu$ were determined and then compared with the observations.

The solution of Eq. (15) with an analytic form of the left side of Eq. (19) is given by

$$
\varphi(\nu)=C e^{-\nu s} \nu^{-4 / 3}
$$

where the parameter $s$, which has the dimensions of time, turns out to be equal to $s=385 \mathrm{~h}$ for the Pleiades cluster. Eq.(20) implies that some part of the flare stars has mean frequencies of less than 0.001 $\mathrm{h}^{-1}$.

This density function $\varphi(\nu)$ is singular at the point $\nu=0$ so that the integral over the entire range of frequencies diverges. This meant that it was impossible to determine the value of $C$ by normalization. Of course, for small $\nu$ up, the real function should behave differently. $T$ hours of observation cannot provide us with any significant information about the flare statistics of stars for which the mean interval between flares exceeds T. Thus, Ambartsumian suggested that the true function should have the form $\varphi(\nu)=C e^{-\nu s} \nu^{-4} / 3 g(\nu)$ where $g(\nu)$ can be taken equal to unity for large $\nu$ and goes rapidly to zero as $\nu \rightarrow 0$. This made it possible, using normalization, to determine the product $N C$ and to estimate the total number of flare stars in the Pleiades cluster with a flare frequency greater than a certain $\nu_{0}$.

This method was later applied by Parsamyan (1980) to flare stars in the Orion association. As in the case of the Pleiades, the data are well fit by Eq.(19) with the constants $a=0.00072$ and $b=2 / 3$. Thus, a solution is obtained in the form of Eq. (20) with $s=1389 \mathrm{~h}$. Parsamian also determined the distribution function of the flare frequency of faint stars in the Pleiades cluster (Parsamian, 2002, Parsamyan, 1980). Here the chronology of the "first flares" was smoothed using the chronology of "third flares":

$$
m_{1}(t)=m_{1}(0)+t \frac{d m_{1}(0)}{d t}+2 t \int_{0}^{t} \frac{m_{3}\left(t^{\prime}\right)}{t^{\prime 2}} d t^{\prime}-2 \int_{0}^{t} \frac{m_{3}\left(t^{\prime}\right)}{t^{\prime}} d t^{\prime}
$$

This expression can be obtained from Eq. (16) through several transformations.

The probability of using chronologies of higher-order flares has been discussed by Arutyunyan (1984), with appropriate expressions given and numerical simulations carried out to establish the efficiency of the method.

\section{Other methods of determination of distribution function of the frequency of flares.}

1) It has been proposed by Mnatsakanyan \& Mirzoyan (1988) rather than determine the distribution function, to solve the problem of predicting the number $n_{k}(t)$ (see Eq. (8)) of flare stars with time. This statement of the problem is based, in particular, on the fact that predicting the numbers $n_{k}(t)$ in the infinite future is equivalent to finding the function $\varphi(\nu)$ :

$$
n_{k}(T) \underset{T \rightarrow \infty}{\rightarrow} \frac{1}{T} \varphi\left(\frac{k}{T}\right)
$$

According to the authors, their solution is practically unuseful for times exceeding $2 T$. Moreover, due to the unavoidable fluctuations of observational data, such predictions limited by smaller time. This method was examined also by Mirzoyan \& Hambarian (1995). It is shown that the chronology of flare star discoveries in the Pleiades cluster and the Orion association can be described satisfactorily by various distribution functions (gamma, binomial, decreasing exponential, and delta) for the mean frequencies of stellar flares. However, it has been found that this is due to the uncertainty in the observationally derived distribution function for the mean frequency of stellar flares. The most likely function is that derived by Ambartsumian, which has a physical basis.

2) Another method has been proposed in Akopian (2003) for determining the distribution function of the flare frequency of randomly flashing objects (flare stars, supernovae). This method essentially involves determining the unknown function through the statistical moments of the distribution. The distribution of the number of observed flares of flare stars, rather than the distribution of the corresponding frequencies, serves as an initial empirical distribution in the problem of determining the flare frequency distribution function. However, the moments of the flare frequency distribution function can be expressed in terms of the corresponding moments of the number of flares. For a sample of flaring objects with a frequency density distribution $\varphi(\nu)$, the moments of the distribution of the number of flares are, respectively, given by

$$
\mu k_{1}=\int \sum_{k=0}^{\infty} k p_{k} \varphi(\nu) d \nu, \quad \mu k_{j}=\int \sum_{k=0}^{\infty}\left(k-\mu k_{1}\right)^{j} p_{k} \varphi(\nu) d \nu, j=2,3,4
$$

where, in particular, $\mu k_{1}$ is the mean number of flares and $\mu k_{2}$ is the variation of in the number of flares. 
Equations (1) and (23) yield some equations relating the moments of the flaring frequency to the moments of the flare number distribution function:

$$
\begin{aligned}
& \mu \nu_{1}=\frac{\mu k_{1}}{t}, \quad \mu \nu_{2}=\frac{\left(\mu k_{2}-\mu k_{1}\right)}{t^{2}}, \quad \mu \nu_{3}=\frac{\left(\mu k_{3}-3 \mu k_{2}+2 \mu k_{1}\right)}{t^{3}}, \\
& \mu \nu_{4}=\frac{\left(\mu k_{4}-6 \mu k_{3}-6 \mu k_{2} \cdot \mu k_{1}+11 \mu k_{2}-6 \mu k_{1}+3 \mu k_{1}^{2}\right)}{t^{4}},
\end{aligned}
$$

where $\mu \nu_{1}=\int \nu \varphi(\nu) d \nu, \quad \mu \nu_{j}=\int\left(\nu-\mu \nu_{1}\right)^{j} \varphi(\nu) d \nu, \quad \mathrm{j}=2,3,4$

Substituting the empirical moments of the distribution of the number of flares into Eq. (24), one can obtain the corresponding empirical moments of the distribution function of the frequency of flares. Thus, the problem is reduced to determining the distribution functions using known distribution moments. This was done using the technique of curve fitting in the Pearson family of distributions by the method of moments. Numerical modelling has shown that the method is quite effective for determining the distribution function of the flare frequency. This method has been applied to the flare stars the Orion association and the Pleiades cluster, solar-type superflare stars.

3) In his first paper on the statistics of flare stars, Ambartsumian (1969) already pointed out the analogy between the statistics of flare stars and the statistics of supernovae (SNe). Here, according to Ambartsumian (1969), it is necessary to treat galaxies as "flaring objects" and replace the term "flare star" with "galaxy" and the concept of "stellar flare" with the concept of "supernova explosion in a galaxy" in the preceding discussions. In this way, a possible derivation by the analogy of the frequency distribution for different sets of galaxies could be examined.

However, despite this similarity, certain differences that owned to the specific features of the observational data, prevent using of the Ambartsumian method in its original form. First, as opposed to the total number of flare stars, the overall number of galaxies in a sample is known in most cases since a sample is constructed by the observer, under the problem that has been set. Second, the duration of the observation time for individual galaxies in the sample is different: in the case of flare stars, it is usually the same (there can be some important exceptions, as in the case of X-ray flare stars). Of course, the data can be cut off to a minimum common observation time, but this leads to a significant reduction of already scant data.

These differences have naturally revealed in the solution of the problem. In this case, we are in need to use censored data and methods for processing such data. In this task, a censored observation is one of a galaxy/star in which not a single SN explosions/ flare observed during the entire observation period. The unknown density distribution of the mean frequency (rate) $\varphi(\nu)$ is equal to the inverse Laplace transform of the survival function (Akopian, 1996):

$$
\varphi(\nu)=L^{-1}\left[F_{1}(T)\right]
$$

where $F_{1}(T)=1-M_{1}(T)$ is the survival or reliability function, and $M_{1}(T)$ is the distribution function of the times of first explosions /flares:

$$
M_{1}(t)=\int_{0}^{t} \frac{m_{1}\left(t^{\prime}\right)}{N} d t^{\prime}
$$

Let us denote the nominal times at which SNe explosions/ flares are detected by $t$. Let $T^{\prime}$ be the time of the first SN explosion/ flare in a given galaxy/star if it has taken place, whereas if no explosion/ flare has not taken place then $T^{\prime}$ is the overall time of the observations of this galaxy/star, and let $n$ be the number of galaxies/stars for which $T^{\prime}>t$ while $k$ is the number of galaxies/stars for which the first SN explosion/flare have been observed at the nominal time $t$. In this case, the estimate of the maximum reasonable survival function $F_{1}(T)$ is the Kaplan-Meier estimator:

$$
F_{1}(T)=\prod^{T}\left(1-\frac{k_{i}}{n_{i}}\right)
$$

with the variation

$$
\operatorname{var}\left[F_{1}(T)\right]=\left[F_{1}(T)\right]^{2} \sum^{T} \frac{k_{i}}{n_{i}\left(n_{i}-k_{i}\right)}
$$

where $\sum$ denotes the sum over all $i$ such that $t<T$. As yet, however, there are insufficient data for the use of these statistical methods, since supernova flares have only been observed in a small number of galaxies so far.

In the practical solution of inverse problems, it is useful to know preliminary information about the nature of the original function. The function $F_{1}(T)$ largely satisfies this condition: it, by definition, is a monotonically decreasing function of $T$ and varies strictly within the range from 1 to 0 . The possibility of estimating the uncertainty (28) of the determination $F_{1}(T)$ is also very important.

This method was used to determine X-ray flares frequency distribution of low-mass young stellar objects of Orion nebula and cloud L1688, which is a part of the complex of dark clouds $\rho$ Oph (Akopian, $2012 \mathrm{a}, \mathrm{b})$. 


\subsection{Long- and short-term change in flare activity}

Long-term change in flare activity due to stellar evolution The presence of systems of different ages makes it possible to trace the evolution of stars, in particular, how the flare activity of stars changes with time? The dependence of the luminosity of the brightest flare star on the age of the system containing these stars was pointed out by Haro (1964): there is a relation between the earliest spectral class of flare stars and the age of the system. Subsequently, Kunkel (1975) and Parsamyan (1976) confirmed the existence of the dependence of the luminosity of the system brightest flare star from the system age.

Later the dependence of the mean luminosity of flare stars of a given system on its age was established by Mirzoyan \& Brutyan (1980) and then confirmed by Mirzoyan \& Brutyan (1980) based on observational data of the nearest systems. It was shown that the observed luminosity function of flare stars is shifted toward lower luminosities as the system ages. Two best-studied subsystems of flare stars of various ages in Orion and Pleiades are compared. It is shown that the observed differences between them are in agreement with the evolutionary status of flare stars, presenting a stage in the evolution of red dwarf stars. It is detected that the mean frequency of flares increases towards low luminosity stars. For the same luminosity stars, the flare activity is lower in older stars. Based on these dependencies, Akopian (1999) has been proposed the method for determining the parameters of the initial luminosity function of flare stars.

The dependence of the maximum possible flare amplitude on luminosity was used by Parsamyan (1976, 1995) to determine the age of a stellar system from observations of the flare stars in it. The essence of this method is as follows: on a "flare amplitude-stellar magnitude" $(\Delta m, m)$ diagram for all the flares of flare stars in a given system the upper envelope of the observed flare amplitudes can be fitted by the straight line:

$$
\Delta m=\kappa m+\Delta m_{0},
$$

whose intersection point with the axis $m$ determines the luminosity of the brightest flare star in the given system. Since the spectral class/luminosity of the brightest flare star in the system depends on the age $T$ of the system, this implies that the slope $\kappa$ of this line also depends on the age of the system. The available data yielded:

$$
\kappa=1.31-0.06 \lg T
$$

This is giving a unique opportunity to find stellar system age if there are flare in the system. The method was adapted to determine the age of single flare stars not included in any system.

Ambartsumian (1969) postulated that during the evolution, the frequency of flares of a star undergoes secular (long-term) changes, the nature of which can be determined by comparing the frequencies of flares in populations of stars of different ages. Knowledge of the corresponding distribution functions allows, in principle, to implement this idea. An attempt of this kind was made in Akopian (2008), where the difference in the obtained functions for Orion and Pleiades flare stars was interpreted as a consequence of evolution.

Short-term change in flare activity due to stellar activity and stellar rotations both axial and orbital. At the foundation of all the methods lies the detection of a departure of observational data from a theoretical Poisson distribution. At present, there are no reasons to reject the hypothesis that the distribution of flares has a random character.

To detect possible cyclicity in the flare activity of flare stars in the Pleiades, Mirzoyan \& Oganyan (1977) divided the entire observational period into two approximately equal parts and calculated the theoretical ratios of the number of flare stars that each exhibit $k$ flares in each interval $\left(n_{k, k}\right)$ to the flare stars number that each exhibit $2 k$ flares over the entire period $\left(n_{2 k}\right)$ :

$$
\frac{n_{k, k}}{n_{2 k}}=\frac{(2 k) !}{2 k^{2 k} k ! k !}
$$

A comparison of theoretical and observed ratios showed that the theoretically expected values are significantly larger than the corresponding observed ones for all valuesk. According to Mirzoyan \& Oganyan (1977) this is consistent with the hypothesis of cyclicity of the flare activity of stars in the Pleiades.

A different approach was used by Parsamian \& Andrews (1996). Flare stars of the Orion association that exhibiting five or more flares were chosen for further study. They divided the entire observational period into two parts and from the observed flare frequency in the first interval, they calculated the theoretically expected number of flares in the second interval and its dispersion. If the theoretically expected number of flares in the second interval differed significantly from the corresponding observed number, the given star was suspected of variable flare activity.

In both methods, the authors were not aimed to define the change point time of flaring activity. Such an attempt was made by in Akopian (1999, 2001), Akopian \& Sargsyan (2002), where a piecewise-stationary Poisson process was involved to describe flare time-sequence of the flare stars of Orion Association and 
Pleiades cluster. Consequently, the following question has been considered: is a sequence of flares a stationary or a piecewise-stationary Poisson process, in which a parameter of the Poisson distribution (the flare frequency) has different values in each time intervals of observations? This approach allows, in principle, to determine the probable change point of the flare activity and to estimate the significance level of the detected changes.

Methods of detecting probable cycles in the flaring activity of flare stars and determining the cycle durations are examined by Akopian (2010). A new method of detecting a cycle of flaring activity was proposed assuming periodic flaring activity (flare frequency) in the form $\nu(t)=v_{0}(1+a \sin \omega t), \quad a>1$. This method was applied to two flare stars of the Pleiades cluster, Ton 91 and Ton 377 (possible member of Hyades) based on data obtained during a long monitoring campaign of the Pleiades region performed in $60-80$ th of the last century.

In these works, the task was set to detect changes in the flare activity of stars, regardless of the mechanism of change. It was believed that, in the likeness of the Sun, stars should have their cycles of activity.

An analysis of the relationship between flare activity and the linear distance of the components of the flare star UV Ceti was carried out by Melikian et al. (2011). It was shown that the flare activity of a star depends on the distance between the components, a higher level of activity corresponds to a smaller distance between the components. This is a manifestation of the periodic variability of the flare frequency due to orbital rotation.

Since the flare frequency can be modulated by a periodic process (axial/orbital rotation of the star), the representation of the flare frequency as a periodic function of time seems to be more reasonable and the flare activity can be considered as a Poisson process with a periodic parameter.

It was recommended Akopian (2015) to use the following function as a periodic function of the flare frequency (parameter) of the Poisson distribution:

$$
\nu(t)=\frac{\nu_{0} \exp \left[k \sin \left(\omega_{0} t+\vartheta\right)\right]}{I_{0}(k)} \quad\left(k \geq 0, \omega_{0} \geq 0,0 \leq \vartheta \leq 2 \pi, \nu_{0}>0\right)
$$

where $k, \omega_{0}, \vartheta, \nu_{0}$ are constants, $I_{0}(k)=\int_{0}^{2 \pi} \exp [k \sin (u)] d u$ - is the modified Bessel function of zeroth order of the first kind.

This function was chosen because it gives different forms of the periodic function depending on the value of $k$, from sinusoid to Dirac comb (Shah) function, which is undoubtedly the advantage of this function. Using this approach, Akopian (2015) suspected the existence of a possible periodicity of the frequency with a period equal to the period of the star's orbital or axial rotation for a few solar-type stars with superflares.

Data from the Kepler orbiting observatory are used in a statistical study of the periodic/cyclical variability of the flare frequency of 76 flare stars Akopian (2019). The corresponding periodic functions of the flare frequency are obtained and the possible periodicity of the flare frequency with a period equal to the rotation period of the star is verified. The periodicities of the flare frequency function turned out to be close to the periods of the axial rotation of the stars. For all stars without exception, the periodicity of the flare frequency has been confirmed. These results show that (a) the flare frequency is modulated by the axial rotation of the stars and (b) structural and physical changes in the active regions of these stars are relatively slow.

\subsection{The spatial distribution of flare stars}

The spatial distribution of stars in young stellar associations and clusters, where dynamical relaxation didn't occur yet, contains valuable information on the physical condition (both of stars and whole system) at their birth-time and subsequent evolution. From this point of view, the study of the spatial distribution of flare stars is of some interest.

Spatial distribution of UV Ceti - type flare stars in the solar vicinity and their origin. In the view of the formation of flare stars in stellar clusters and associations, the existence of UV Ceti-type stars in the vicinity of the Sun can have two possible explanations. According to the first hypothesis (Ambartsumian, 1957), these stars were formed in a system that still exists. In other words, UV Ceti-type stars in the vicinity of the Sun constitute a physical system at present. The second explanation (Herbig, 1962, Oskanyan, 1964), assumes that UV Ceti type stars were formed in systems now decayed and appeared in the vicinity of the Sun accidentally, as a result of the "parent" systems decay.

The solution to the problem can be obtained by determining the spatial distribution of stars. If it is uniform, then the second hypothesis is most likely true. If the spatial density of stars abruptly drops off at some distance (the edge of the stars cluster), then the first hypothesis is true. The first considerations Arakelian (1970), Garibdzhanian (1976) of the problem by BAO astronomers, led to a conclusion in favour 
of the first explanation. The further study of the problem Mirzoyan et al. (1988) did not confirm the existence of a system of UV Ceti-type stars around the Sun. As became clear, that conclusion had been obtained as a result of an overestimate of the expected flare numbers produced by UV Ceti type stars in the galactic star field during photographic observations of various regions of the sky.

This result can be regarded as important evidence in favour of the hypothesis that UV Ceti type stars in the vicinity of the Sun, and in general, all flare stars of the galactic field, are formed in stellar clusters and associations. Consequently, there is the common origin of the flare stars in clusters/ associations and the UV Ceti type flare stars in the vicinity of the Sun. This allows supposing that they constitute a single class of stars having the common property of flare activity Mirzoyan (1981). Many parameters of flare stars of these two varieties differ very little, while the important differences between them can be successfully explained by differences in age, e.g. mean luminosity of flare stars of a system.

Spatial distribution of the Pleiades flare stars. The study of the distribution of flare stars in parent systems is of great importance for the problem of stellar evolution. Such a study carried out by Mirzoyan \& Mnatsakanian (1971) for the Pleiades showed that flare stars are almost completely absent in the central part of the system. The radius of this cavity is $1.4 \mathrm{pc}$. The partial density of flare stars reaches a maximum at a distance of $1.5 \mathrm{pc}$ from the centre of the system; then it decreases faster than $\sim r^{-2}$. The solution of the Abel equation was obtained by a method developed by Mnatsakanyan (1969), based on the well-known Plummer's method. The method gives a solution, which is a kind of average of all solutions that can be obtained using Plummer's method.

It has been supposed that the distribution of the flare stars in the system is a spheric-symmetrical one, the centre of the system being the geometrical centre of the known flare stars. It is almost coinciding with Alcyone. However, later studies of the problem didn't Mirzoyan (1981) confirm the results of the first work. The cavity disappeared when the number of discovered flare stars increased.

A study of the surface density of flare stars in the Pleiades by Chavushian et al. (1999a, 2004) led to the discovery of a density deficit at a distance of $3.2 \mathrm{pc}$ from the centre of the cluster. The same feature was revealed in the spatial distribution of these stars. For the construction of flare stars spatial distribution, the one-dimensional distribution was constructed from observed surface distribution. It was shown that it is necessary for reliable construction of the one-dimensional distribution to solve Abel's equation. On its basis, the dependence of real space density distribution of flare stars on the distance from the cluster's centre is determined. As a result, $0.5 \mathrm{pc}(2.8 \mathrm{pc} \leq R \leq 3.5 \mathrm{pc})$ wide spherical layer with the deficit of flare stars was revealed. Preliminary consideration of the problem with new astrometric data from Gaia does not in contradiction with the obtained result.

\subsection{Recent developments and possible applications of methods}

Over the last decade, interest in flare stars and related objects has been renewed and increased sharply. Cosmic telescope research, modern observations on ground-based telescopes have shown that flare stars are also characterized by other manifestations of activity. X-ray and ultraviolet radiation were detected, indicating the presence of powerful corona and chromospheres in these stars. Flares have been directly recorded in a wide range of wavelengths - from hard X-rays and far-ultraviolet radiation to microwave radio emission. Cold spots have been found that manifest themselves in slow variations in the star's brightness with small amplitudes, and long-term cycles of activity have been discovered, similar to the 11-year solar cycle. This is mainly because of observations with the Kepler space telescope intended to search for exoplanets. Because of its unique characteristics, observations by the Kepler telescope simultaneously provide valuable and uniform data on variable stars and variable phenomena of almost all kinds, including flare stars and flare phenomena. A comparatively long series of almost continuous and uniform observations with high sensitivity, a large field, and a huge number of simultaneously observed objects facilitate comprehensive (both statistical and physical) studies of flare stars and flare phenomena. The complexity of these observations shows up not only and not so much in the number of characteristics of the data, as in the fact that data on very important physical parameters closely related to the variability of stars are obtained simultaneously. In the case of flare stars, these data can be used for the following:

- to obtain a light curve over a fairly long time, which in principle makes it possible to study the variation of flare activity during the stellar activity cycle (analogous to the solar activity cycle),

- estimate the rotation period and spottedness of stars, and, accordingly, the sizes and energy characteristics of active regions, and

- detect differential rotation and the migration of active regions, and study their relationship to flare activity.

This makes it possible to carry out more complete physical and statistical studies of the periodic/cyclical variability of the observed frequency of flares of flare stars, as well as to detect flares and flare objects of new types, in particular, solar-type stars with superflares.

In this process, the methods and approaches developed at the BAO for the study of flare stars will certainly be very useful. 


\section{References}

Akopian A. A., 1996, Astrophysics, 39, 331

Akopian A. A., 1998, Astrophysics, 41, 47

Akopian A. A., 1999, Astrophysics, 42, 419

Akopian A. A., 2001, Astrophysics, 44, 106

Akopian A. A., 2003, Astrophysics, 46, 58

Akopian A. A., 2008, Astrophysics, 51, 48

Akopian A. A., 2010, Astrophysics, 53, 544

Akopian A. A., 2012a, Astrophysics, 55, 81

Akopian A. A., 2012b, Astrophysics, 55, 505

Akopian A. A., 2013, Astrophysics, 56, 488

Akopian A. A., 2015, Astrophysics, 58, 62

Akopian A. A., 2017, Astrophysics, 60, 118

Akopian A. A., 2018, Communications of the Byurakan Astrophysical Observatory, 65, 65

Akopian A. A., 2019, Astrophysics, 62, 518

Akopian A. A., Sargsyan L. A., 2002, Astrophysics, 45, 20

Akopyan A. A., 1995, Astrophysics, 38, 157

Ambartsumian V. A., 1947, The evolution of stars and astrophysics. Akad Nauk Arm SSR, Erevan

Ambartsumian V. A., 1949, Astronomicheskii Zhurnal, 26, 3

Ambartsumian V. A., 1954, Communications of the Byurakan Astrophysical Observatory, 13, 3

Ambartsumian V. A., 1957, in "Non-stable stars", ed. Herbig, G.H.. p. 177

Ambartsumian V. A., 1969, Stars, Nebulae, and Galaxies, Izd-vo. Akad Nauk Arm. SSR,, p. 283

Ambartsumian R. V., 1998, Editors addendum in "A life in astrophysics : selected papers of Viktor A. Ambartsumian", Publisher: New York, NY: Allerton Press,,

Ambartsumian V. A., Mirzoian L. V., 1975, in Proc. IAU Symp. 67 "Variable Stars and Stellar Evolution" ed. Sherwood, V. E. and Plaut, L., pp 3-14

Ambartsumian V. A., Mirzoyan L. V., 1971, in IAU Colloq. 15: New Directions and New Frontiers in Variable Star Research. p. 98

Ambartsumjan V. A., 1988, Scientific Works. Vol.3. Publisher, Address

Ambartsumyan V. A., 1970, Astrophysics, 6, 11

Ambartsumyan V. A., 1978, Astrophysics, 14, 209

Ambartsumyan V. A., Mirzoyan L. V., Parsamyan E. S., Chavushyan O. S., Erastova L. K., 1970, Astrophysics, 6, 1

Ambartsumyan V. A., Mirzoyan A. V., Parsamyan É. S., Chavushyan O. S., Erastova L. K., 1971, Astrophysics, 7, 189

Ambartsumyan V. A., Mirzoyan L. V., Parsamyan É. S., Chavushyan O. S., Erastova L. K., Kazaryan É. S., Oganyan G. B., 1972, Astrophysics, 8, 287

Ambartsumyan V. A., Mirzoyan L. V., Parsamyan É. S., Chavushyan O. S., Erastova L. K., Kazaryan É. S., Oganyan G. B., Yankovich I. I., 1973, Astrophysics, 9, 267

Arakelian M. A., 1970, Communications of the Byurakan Astrophysical Observatory, 41, 56

Arutyunyan G. A., 1984, Astrophysics, 21, 450

Bohning D., 2010, Scandinavian Journal of Statistics, 37, 221

Chavushian H. S., 1979, PhD thesis, Byurakan Astrophysical Observatory, Armenia.

Chavushian H. S., Broutian G. H., Oskanian A. V., 1999a, Astrophysics, 42, 264

Chavushian H. S., Oskanian A. V., Broutian G. H., 1999b, Astrophysics, 42, 404

Chavushian H. S., Pikichian H. V., Oskanian A. V., Broutian G. H., 2004, Astrophysics, 47, 313 
Garibdzhanian A. T., 1976, Communications of the Byurakan Astrophysical Observatory, 49, 63

Haro G., 1957, "Non-stable stars", proceedings from IAU Symposium N. 3, ed. Herbig, G. H., p. 26

Haro G., 1964, "The Galaxy and the Magellanic Clouds", Proceedings of the IAU Symposium N. 20, ed.Frank, J. K., p. 26

Herbig G. H., 1962, Proceedings of a Symposium on Stellar Evolution, held in La Plata, ed. Sahade, J., p. 23

Kunkel W. E., 1975, "Variable Stars and Stellar Evolution"; Proceedings of the Symposium, Moscow, ed. Sherwood, V. E. and Plaut, L., p. 67

Melikian N. D., Tamazian V. S., Samsonyan A. L., 2011, Astrophysics, 54, 469

Mirzoyan L. V., 1981, Izvestiya Akademiya Nauk Armyanskoi, 16, 126

Mirzoyan A. V., 1993, Astrophysics, 36, 170

Mirzoyan L. V., Ambaryan V. V., 1988, Astrophysics, 28, 220

Mirzoyan L. V., Brutyan G. A., 1980, Astrophysics, 16, 67

Mirzoyan A. L., Hambarian V. V., 1995, Astrophysics, 38, 11

Mirzoyan L. V., Mnatsakanian M. A., 1971, Information Bulletin on Variable Stars, 604, 1

Mirzoyan A. L., Mnatsakanian M. A., 1990, "Flare Stars in Star Clusters, Associations, and the Solar Vicinity". Proceedings of the 137th. Symposium of the IAU, Armenia, ed. Mirzoian, L. V. and Pettersen, B. R. and Tsvetkov, M. K., p. 77

Mirzoyan L. V., Oganyan G. B., 1977, Astrophysics, 13, 333

Mirzoyan L. V., Chavushyan O. S., Erastova L. K., Oganyan G. B., Melikyan N. D., Natsvlishvili R. S., Tsvetkov M. K., 1977, Astrophysics, 13, 105

Mirzoyan L. V., Ambaryan V. V., Garibdzhanyan A. T., Mirzoyan A. L., 1988, Astrophysics, 29, 430

Mnatsakanyan M. A., 1969, Doklady Akademiia Nauk ArmSSR, 49, 33

Mnatsakanyan M. A., 1986, Astrofizika, 24, 621

Mnatsakanyan M. A., Mirzoyan A. L., 1988, Astrophysics, 29, 423

Oskanyan V. S., 1964, Publ. Astron. Observ. Beograd, 10

Oskanyan V. S., Terebizh V. Y., 1971, Astrophysics, 7, 48

Parsamian E. S., 2002, Astrophysics, 45, 15

Parsamian E. S., Andrews A. D., 1996, Irish Astronomical Journal, 23, 183

Parsamyan É. S., 1976, Astrophysics, 12, 145

Parsamyan É. S., 1980, Astrophysics, 16, 391

Parsamyan E. S., 1995, Astrophysics, 38, 206 